\title{
Water-Mediated Dimerization of Ubiquitin Ions Captured by Cryogenic Ion Mobility-Mass Spectrometry
}

Kelly A. Servage,${ }^{\dagger}$ Joshua A. Silveira, ${ }^{\dagger}$ Kyle L. Fort, ${ }^{\dagger}$ David E. Clemmer, ${ }^{\ddagger}$ and David H. Russell ${ }^{\dagger *}$

${ }^{\dagger}$ Department of Chemistry, Texas A\&M University, College Station, Texas 77843, United States

${ }^{\ddagger}$ Department of Chemistry, Indiana University, Bloomington, Indiana 47405, United States

\section{Table of Contents}

Page No.

Experimental notes for ion mobility arrival time distributions $\quad$ S2

Figure S1 S3

Figure S2 S3

$\begin{array}{ll}\text { Figure S3 S4 } & \text { S4 }\end{array}$ 


\section{Experimental Notes}

\section{Ion Mobility Arrival Time Distributions}

Due to difficulties associated with converting arrival time distributions (ATD) to collision cross sections (CCS) at $80 \mathrm{~K}$, all mobility plots of ubiquitin ions shown here are reported in arrival time. Complications obtaining accurate CCS measurements at low temperatures both experimentally and computationally have been shown previously. ${ }^{1-3}$ Because the majority of IMMS experiments are conducted under ambient conditions $(300 \mathrm{~K})$, data on CCS values of biomolecules at $80 \mathrm{~K}$ are limited, making calibration difficult. Additionally, difficulties associated with generating $1 / \mathrm{V}$ plots of hydrated ions exist due to the weakly bound state of most of these species. There is a narrow range of field strength that can be operated under before inducing dissociation of hydrated ions in the IM drift tube and potentially losing the ions of interest. ${ }^{4} \mathrm{We}$ are currently working on addressing these issues to ultimately report CCS measurements of hydrated ions at $80 \mathrm{~K}$, but currently these problems are not solved. For this reason, mobility plots have been shown in arrival time and additional evidence supporting our assignment of peaks as monomer and dimer ions is reported below.

\section{References}

1) Bleiholder, C.; Wyttenbach, T.; Bowers, M. T. Int. J. Mass Spectrom. 2011, 308, 1-10.

2) Bleiholder, C.; Contreras, S.; Bowers, M. T. Int. J. Mass Spectrom. 2013, 354-355, 275-280.

3) Mesleh, M.F.; Hunter, J. M.; Shvartsburg, A. A.; Schatz, G. C.; Jarrold, M. F. J. Phys. Chem. 1996, 100, 16082-16086.

4) Silveira, J. A.; Servage, K. A.; Gamage, C. M.; Russell, D. H. J. Phys. Chem. A 2013, 117, 953961. 

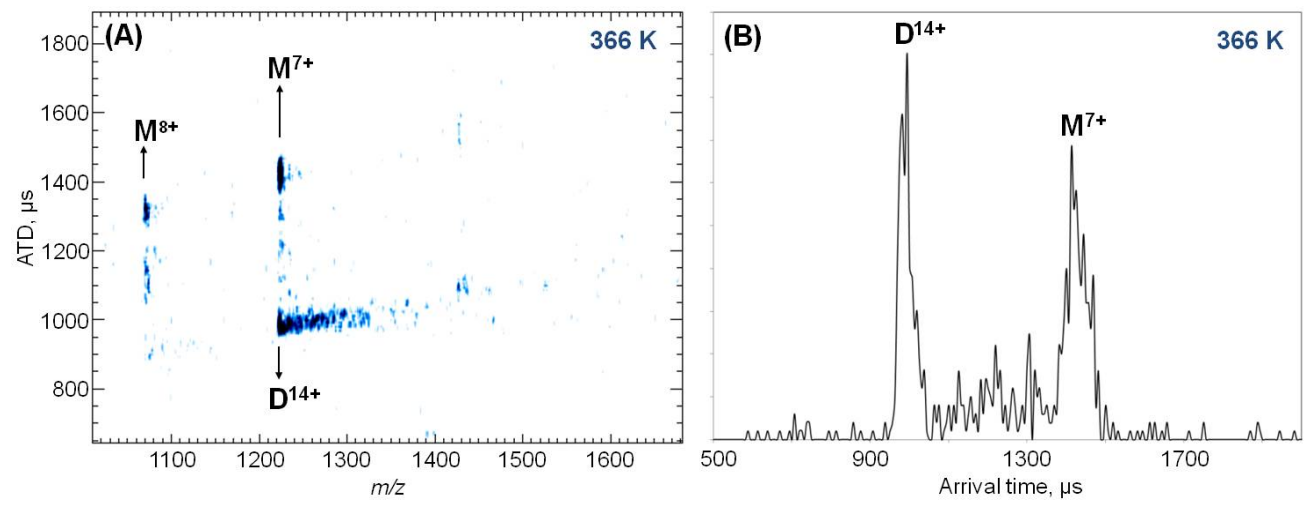

Figure S1. (A) 2-D contour plot of ATD versus $\mathrm{m} / \mathrm{z}$ for ubiquitin ions collected at a concentration of $75 \mu \mathrm{M}$. (B) Mass-selected ATD of the +7 charge state extracted from the data shown in (A). Solution conditions are the same as those shown in Figure 2 (pure water with $0.1 \%$ formic acid). Increasing the concentration to $75 \mu \mathrm{M}$ results in the observed increase in abundance of the dimer ion population relative to that of the monomer, consistent with our assignment of peaks.

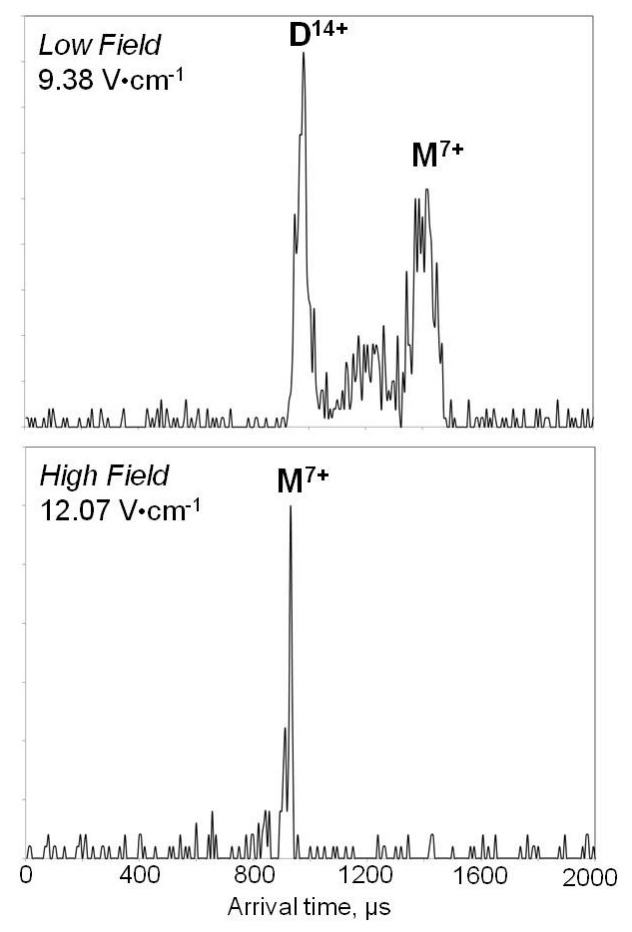

Figure S2. Mass-selected ATDs of the +7 charge state of ubiquitin ions collected under low field strength conditions across the drift tube $\left(9.38 \mathrm{~V} \cdot \mathrm{cm}^{-1}\right)$ compared to high field $\left(12.07 \mathrm{~V} \cdot \mathrm{cm}^{-1}\right)$. The "low-field limit" for the cryo-IM-MS instrument was previously established and it was shown that at higher fields $\left(>10.4 \mathrm{~V} \cdot \mathrm{cm}^{-1}\right)$, collision induced dissociation resulted in the dissociation of weakly bound species during the IM measurement. ${ }^{4}$ The data shown here is consistent with this finding, as the ATD of the monomer ion expectedly shifts to shorter drift time with increased field strength and the peak assigned to the dimer ion disappears completely. By increasing the field strength across the drift tube, the effective ion temperature of the dimer is increased and CID of the weakly bound species results in dissociation of the ion. 


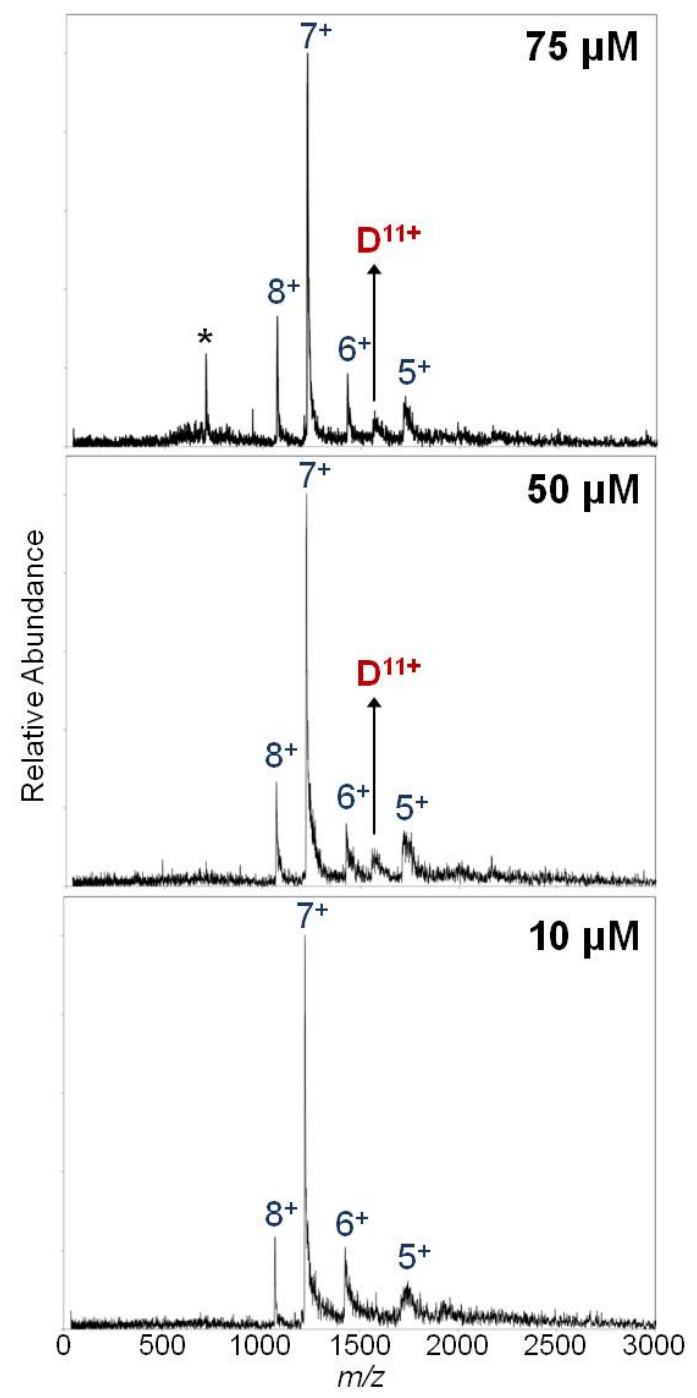

Figure S3. Mass spectra of ubiquitin ions collected at concentrations of $10 \mu \mathrm{M}, 50 \mu \mathrm{M}$, and 75 $\mu \mathrm{M}$. All solutions were prepared in pure water with $0.1 \%$ formic acid to produce the "native" charge state distribution for ubiquitin, centered on the $[\mathrm{M}+7 \mathrm{H}]^{7+}$ ion. The spectra clearly show the presence of an asymmetric dimer, $[2 \mathrm{M}+11 \mathrm{H}]^{11+}$, in low abundance at increased concentrations $(50 \mu \mathrm{M}$ and $75 \mu \mathrm{M})$. The formation of asymmetric dimers is not unexpected at high concentrations and supports the notion that noncovalently bound dimers of ubiquitin are being preserved in the experiment. The peak labeled with an asterisk is an unidentified contaminant ion. 